https://doi.org/10.15407/ujpe65.6.521

V.V. DATSYUK, O.R. PAVLYNIUK

Taras Shevchenko National University of Kyiv, Faculty of Physics

(Kyiv 01601, Ukraine; e-mail: datsyuk@univ.kiev.ua)

\title{
THE ROLE OF SURFACE-CHARGE TRANSPORT IN ELECTROHYDRODYNAMICS AND ELECTROMECHANICS OF A DIELECTRIC SPHERE
}

\begin{abstract}
To simulate the electrokinetic processes in weakly-conducting dielectric media, the TaylorMelcher leaky-dielectric model is widely used, though its applicability conditions are unknown. To define them, the electric-potential distributions inside and outside a dielectric sphere placed in an electric field are determined, by assuming the sphere and the environment are weakly conducting and by considering the electric and diffusion interfacial currents and the surface-charge decay. Earlier, an electric-field characteristic of a dielectric sphere, for example, the real part of the Clausius-Mossotti factor found for a direct current (DC) field was commonly thought to be a single-valued function of two parameters, the conductivities of the sphere and the environment. Now, it depends on a larger number of parameters and, in the dc case, can range from the perfect-dielectric to perfect-conductor values even for a particle of a good insulator. Using the proposed theory, a variety of the experimental results on the electrohydrodynamic (EHD) fluid circulation and dielectrophoretic (DEP) motion of microparticles in the dielectric drops are explained for the first time or in a new way. The dielectrophoretic inflection and cross-over frequencies are defined allowing for the decay of the surface charge. A dependence of the effective conductivity of a sphere on the angular field distribution is predicted for the first time.

Ke ywords: dielectrophoresis, electrohydrodynamics, leaky-dielectric model.
\end{abstract}

\section{Introduction}

A new technology of the electric-field manipulation, characterization, and separation of cells, microorganisms, macromolecules, DNA, viruses, and dielectric microparticles was proposed and successfully developed in the second half of the 20th century [110]. During the past decade, a branch of this technology was focused on the manipulation by microparticles on the surface of a dielectric droplet [11-21]. In particular, the attention was paid to the formation of structures of microparticles at the surface of a millimeter-sized drop $[14,15,20]$. It is of interest that a silicone-oil drop covered by chains of highly conducting microparticles was transformed into a perfect dielectric in one experiment [14], but into a perfect conductor in another one [20]. We found no explanation of this feature in the literature. Similarly, the applicability conditions for two different approaches of the electromagnetic theory have not yet defined. On the one hand, the potential distribution for a perfect-

(C) V.V. DATSYUK, O.R. PAVLYNIUK, 2020

ISSN 2071-0194. Ukr. J. Phys. 2020. Vol. 65, No. 6 dielectric sphere in a uniform electric field $\mathbf{E}$ is found, maybe, in each textbook on the electromagnetic theory and optics including modern ones [2, 6, 22]. For a perfect dielectric sphere with the permittivity $K_{2}$ in a perfect dielectric with the permittivity $K_{1}$, the potential $V_{\text {pd }}$ is presented in Table, where $r$ is the radial coordinate in the spherical coordinate system with the origin at the center of the sphere and the $z$ axis parallel to $\mathbf{E}$. A perfect dielectric is a substance that has no conductivity.

On the other hand, a sphere of a nonideal dielectric can be treated as conductive, which gives $V_{\text {ld }}$, the potential of the model [21,23-25]. This model is commonly applied to simulate the electrohydrodynamic (EHD) flows inside and outside a dielectric drop in a uniform electric field. This approach was introduced by Taylor [23] for the dc case and was generalized by Torza, Cox, and Mason [26] (TCM) for the alternating current $(\mathrm{AC})$ case. The "electric" part of the leaky-dielectric model is of use in the theory of dielectrophoresis, since the DEP force is proportional to the real part $\beta$ of the Clausius-Mossotti factor $f_{\mathrm{CM}}$. This 
factor is found from Table 1 as the term before the multiplier $(a / r)^{3}$ in the square brackets. In the theory of dielectrophoresis, the Clausius-Mossotti factor $f_{\mathrm{CM}}$ plays an important role. The product of this factor and $a^{3}$, where $a$ is the radius, is the polarizability $\alpha$ of the sphere. In particular, $f_{\mathrm{CM}}$ determines the direction and absolute value of the DEP force acting on a particle in a homogeneous medium. The DEP force acting on a particle attached to the drop interface depends on the product $\beta(2+\beta) \beta^{\prime}[18,20]$, where $\beta$ and $\beta^{\prime}$ are the real parts of the Clausius-Mossotti factors of the drop and particle in the ambient liquid, respectively. The values of $\beta$ and $\beta^{\prime}$ can readily be found using the "electric" part of the TaylorMelcher leaky-dielectric model [23, 24, 26]. Here, we will establish, for the first time to the best of our knowledge, its applicability limits and define the basic electric properties of a weakly conductive sphere beyond them.

To understand the distinction of this study, let us address the following question: What potential should be used in practice to determine the polarization of a dielectric sphere, $V_{\mathrm{ld}}$ or $V_{\mathrm{pd}}$ ? At first glance, the Taylor-Melcher leaky-dielectric model is preferable, as it involves the conductivities $\sigma_{1}$ and $\sigma_{2}$ of the ambient medium and drop, respectively, neglected in the alternative model that considers the system of ideal dielectrics. However, there are no dielectric constants $K_{1}$ and $K_{2}$ in the formulas for $V$ in the leaky-dielectric, or conductive [2], model. Therefore, the Taylor-Melcher model is not more general than the perfect-dielectric one. Indeed, the first line in Table does not follow from the second line by setting $\sigma_{1}=\sigma_{2}=0$. Secondly, both $V_{\mathrm{ld}}$ and $V_{\mathrm{pd}}$ depend

Potential distributions for dc perfect-dielectric, leaky-dielectric, perfect-conductor, and ac leaky-dielectric models

\begin{tabular}{|c|c|c|}
\hline$V$ & $r \leq a$ & $r \geq a$ \\
\hline$V_{\mathrm{pd}}$ & $-\frac{3 K_{1}}{2 K_{1}+K_{2}} z E$ & {$\left[\frac{K_{2}-K_{1}}{2 K_{1}+K_{2}}\left(\frac{a}{r}\right)^{3}-1\right] z E$} \\
$V_{\mathrm{ld}}$ & $-\frac{3 \sigma_{1}}{2 \sigma_{1}+\sigma_{2}} z E$ & {$\left[\frac{\sigma_{2}-\sigma_{1}}{2 \sigma_{1}+\sigma_{2}}\left(\frac{a}{r}\right)^{3}-1\right] z E$} \\
$V_{\mathrm{pc}}$ & 0 & {$\left[\left(\frac{a}{r}\right)^{3}-1\right] z E$} \\
$V_{\mathrm{ac}}$ & $-\frac{3 \varepsilon_{1}}{2 \varepsilon_{1}+\varepsilon_{2}} z E$ & {$\left[\frac{\varepsilon_{2}-\varepsilon_{1}}{2 \varepsilon_{1}+\varepsilon_{2}}\left(\frac{a}{r}\right)^{3}-1\right] z E$} \\
\hline
\end{tabular}

on the material parameters, whose numbers are the same: $\sigma_{1}, \sigma_{2}$ and $K_{1}, K_{2}$, respectively.

The situation is seemingly clear, if an ac field is applied. For a time dependence of the form $e^{-i \omega t}$, the permittivities $K$ are commonly replaced with $[2$, $7,22,27]$

$\varepsilon=K+i \sigma /\left(\omega \varepsilon_{0}\right)$,

giving the potential $V_{\mathrm{ac}}$ [2]. Here, $\varepsilon_{0}$ is the electric constant. The potential $V_{\mathrm{ac}} \rightarrow V_{\mathrm{ld}}$ at low frequencies, where $\omega \tau_{\beta} \rightarrow 0$, while $V_{\mathrm{ac}} \rightarrow V_{\mathrm{pd}}$ at high frequencies, where $\omega \tau_{\beta} \rightarrow \infty[2,7,27,28]$. Here, $\tau_{\beta}=\varepsilon_{0}\left(K_{2}+\right.$ $\left.+2 K_{1}\right) /\left(\sigma_{2}+2 \sigma_{1}\right)$ is the Maxwell-Wagner relaxation time $[2,6,21,27]$.

Here, we introduce a surface-charge decay time $\tau_{\alpha}$ which, in particular, depends on the surface diffusion coefficient. Our model gives the potential $V=V_{\mathrm{pd}}$ at high frequencies, indeed. However, at low frequencies or in the dc case, the potential can be presented in the form of a linear combination of $V_{\mathrm{pd}}$ and $V_{\text {ld }}$ giving $V=V_{\mathrm{pd}}$ at $\tau_{\beta} \gg \tau_{\alpha}$ and $V=V_{\mathrm{ld}}$ at $\tau_{\beta} \ll \tau_{\alpha}$. Thus, there is a significant qualitative distinction between our model and the Taylor-Melcher leaky-dielectric one.

Recently, many authors [1,3-7, 9, 18,27,29-38] took the surface conductance $\kappa$ into account using the conductivity $\sigma$ of a sphere of radius $a$,

$\sigma=\sigma_{\mathrm{b}}+2 \frac{\kappa}{a}$

instead of the bulk conductivity $\sigma_{\mathrm{b}}$. This formula was likely to be derived for the first time by Schwan in a study of the electric properties of cells [39], see also [29]. The same formula (2) was also obtained for a bare sphere in a uniform field [40]. A theory of dielectric dispersion of colloidal particles in electrolyte solutions [41] accounts for not only the surface conductivity, but also the surface diffusion. Despite the long history, it is not yet the common practice to take the surface effects into account. Equation (2) explained the size dependence of the motion of micron and submicron particles observed in experiments $[30,31,37,42-44]$ in a very broad range of the electrolyte conductivities. These experimental data were discussed within the models $[5,31,34,38,42,43,45,46]$ considering diverse electrokinetic processes in the Stern and diffuse layers $[33,47]$. The theoretical studies of the polarization of 
the double layer of spherical particles were started by Maxwell and Wagner. Their ideas were being developed since the 1960s (see Refs. [48, 49] and references therein). For example, in Ref. [50] Dukhin and Shilov set forth their theory of giant low-frequency dispersion of the permittivity for dispersed media of spherical particles and particles of other shapes. They considered three possible mechanisms of ion transport in an electrolyte through the migration in an electric field, diffusion, and ion motion caused by the fluid convection. In the dielectricrelaxation spectroscopy $[3,32,33,48,51]$, the relaxation associated with the diffusion of ions at a surface was called the low-frequency double layer relaxation or $\alpha$ relaxation. Simultaneously, the relaxation characterized with the Maxwell-Wagner (or Maxwell-WagnerO'Konski $[3,32,33]$ ) relaxation time was called the high-frequency relaxation or $\beta$-relaxation. The previous studies [1, 5, 29, 31, 34, 38, 42, 43, 45, 46, 52, 53] were focused mostly on the surface constitution and mechanisms of the interfacial current. In particular, the paper by Basuray, Wei, and Chang [45] was focused on the calculation of the cross-over frequency $\omega_{\mathrm{c}}$, at which the induced dipole reverses the direction relative to the bulk field and $\beta\left(\omega_{\mathrm{c}}\right)=0$. The authors considered three limiting cases: a) large Debye layer asymptote, b) thin Debye layer asymptote, and c) polar charging asymptote, when the charging occurs only at the poles through the field focusing around the insulated colloid. Their theory allowed for the following mechanisms affecting the ac polarization: conductivity gradient between the layer and the bulk (which accumulates a space charge) due to the surface charge attraction, tangential diffusion/current, and normal electric displacement. The cross-over frequencies calculated without using the empirical parameters were noted to collapse with the experimental data for latex nanocolloids of various sizes in different electrolytes with widely ranging ionic strengths.

Our study differs from those cited above in the task statement. We consider $\kappa$ and the surface-diffusion coefficient $D_{12}$ as given parameters and investigate their influence on the DEP and EHD motions of microparticles and liquids. Thereby, we follow Taylor [23] and Melcher [24], Torza, Cox, and Mason [26], who determined the polarization of an entire sphere without considering the properties of its double layer. This, in fact, phenomenological approach can be applied successfully to the modeling of com- plex systems with surfaces covered by thin films or chains of highly-conducting particles.

In the rest of this paper, we will present a general model of electric properties of a weakly conductive dielectric sphere, compare it with other theories, and offer new explanations for some experiments [18,43,54]. The principal distinction of our model from the leakydielectric one $[23,24,26]$ consists in the account for diffusion currents. However, even the known process of surface conductivity requires a careful consideration. Thus, we will show that Eq. (2) is applicable, only if the external field is uniform.

\section{Methods}

In this paper, we solve a problem of the electrostatics for a simple spherical geometry, when the general solution of the Laplace equation is known. Therefore, our task reduces to the setting and solution of a system of boundary conditions. There are two usual boundary conditions for the electric potential and the normal projection of the electric field vector. An additional balance equation is introduced to determine the surface charge. After the potential distribution is defined, the common approaches $[18,23,26]$ can be applied to calculate a drop deformation and the fluid flow and to predict the motion of microparticles attached to the surface.

We adopt the Maxwell stress tensor in the form by Torza et al. [26] to calculate the surface force at the interface of a dielectric sphere. In particular, the tangential stress that generates a fluid flow was defined as follows

$S_{\theta}=-s_{\mathrm{p}} E_{2} \sin \theta \cos \theta$,

where $\theta$ is the polar angle, $s_{\mathrm{p}}$ is the surface charge at the north pole of the sphere, where $\theta=0$, and $E_{2}$ is the absolute value of the electric field vector at the inner surface.

\section{Polarization of a Weakly Conductive Sphere in the Electric Field}

\subsection{Theoretical model}

Consider a conductive sphere (medium 2) embedded in conductive medium 1 in a uniform electric field parallel to the $z$ axis. The superposition of the external field with that of the polarized sphere is described by the potentials $V_{1}=-E z+\left(C_{1} / r^{2}\right) \cos \theta$ at $r \geq a$ 
and $V_{2}=C_{2} r \cos \theta$ at $r \leq a$. The coefficients $C_{1}$ and $C_{2}$ are determined from the following boundary conditions:

$V_{1}=V_{2}, \quad$ at $\quad r=a ;$

$-K_{1} \frac{\partial V_{1}}{\partial r}+K_{2} \frac{\partial V_{2}}{\partial r}=\frac{s}{\varepsilon_{0}}, \quad$ at $\quad r=a$,

where $s$ is the surface charge which should be determined from a charge balance equation.

By using Eqs. (4) and (5), the potentials outside and inside the sphere and the surface charge are found to be

$$
\begin{aligned}
& V_{1}=-z E+\frac{z}{K_{2}+2 K_{1}}\left[\left(K_{2}-K_{1}\right) E+\frac{s_{\mathrm{p}}}{\varepsilon_{0}}\right]\left(\frac{a}{r}\right)^{3}, \\
& V_{2}=\frac{z}{K_{2}+2 K_{1}}\left(-3 K_{1} E+\frac{s_{\mathrm{p}}}{\varepsilon_{0}}\right), \quad s=s_{\mathrm{p}} \cos \theta .
\end{aligned}
$$

In order to determine $s_{\mathrm{p}}$, the charge balance at the surface should be taken into account in addition to the boundary conditions $(4,5)$.

Let us consider a variation of the surface charge in the area between the arcs at the sphere surface: $\theta=\theta_{0}, \theta=\theta_{0}+\mathrm{d} \theta, \phi=\phi_{0}$, and $\phi=$ $=\phi_{0}+\mathrm{d} \phi$, where the prefix $\mathrm{d}$ denotes an infinitesimal increment, and $\phi$ is the azimuthal angle. The charge that exits this area per unit time in the direction of the $\mathbf{r}$ vector is equal to $\left[j_{1 r}\left(a, \theta_{0}\right)-\right.$ $\left.-j_{2 r}\left(a, \theta_{0}\right)+s\left(a, \theta_{0}\right) / \tau_{\mathrm{s}}\right] a^{2} \sin \theta_{0} \mathrm{~d} \theta \mathrm{d} \phi$. Here, $\tau_{\mathrm{s}}^{-1}$ is the surface-charge decay rate that accounts for the charge emission from the surface due to the volume diffusion, steaming, or ejection of a charged liquid, etc. The charge that exits the area per unit time through the $\operatorname{arcs} \theta=\theta_{0}$ and $\theta=\theta_{0}+\mathrm{d} \theta$ is $\left[\sin \left(\theta_{0}+\mathrm{d} \theta\right) i_{\theta}\left(\theta_{0}+\mathrm{d} \theta, \phi_{0}\right)-\sin \left(\theta_{0}\right) i_{\theta}\left(\theta_{0}, \phi_{0}\right)\right] a \mathrm{~d} \phi=$ $=\frac{\partial}{\partial \theta}\left[\sin \left(\theta_{0}\right) i_{\theta}\left(\theta_{0}, \phi_{0}\right)\right] a \mathrm{~d} \theta \mathrm{d} \phi$. Finally, there can be a surface-charge flow in the direction of the unit vector $\mathbf{e}_{\phi}$, that is $\left[i_{\phi}\left(\theta_{0}, \phi_{0}+\mathrm{d} \phi\right)-i_{\phi}\left(\theta_{0}, \phi_{0}\right)\right] a \mathrm{~d} \theta=$ $=\frac{\partial}{\partial \phi} i_{\phi}\left(\theta_{0}, \phi_{0}\right) a \mathrm{~d} \theta \mathrm{d} \phi$. By summarizing all the currents and dividing the sum by $a^{2} \sin \theta_{0} \mathrm{~d} \theta \mathrm{d} \phi$, we get

$\frac{\partial s}{\partial t}=-j_{1 r}+j_{2 r}-\frac{s}{\tau_{\mathrm{s}}}-\frac{1}{a \sin \theta}\left[\frac{\partial}{\partial \theta}\left(\sin \theta i_{\theta}\right)+\frac{\partial}{\partial \phi} i_{\phi}\right]$.

In this model, the volume current densities are determined by the Ohm's law:

$\mathbf{j}_{i}=\sigma_{i} \mathbf{E}_{i}$.
However, we take the surface diffusion into account in the definition of a surface current density:

$i_{\theta}=\kappa E_{1 \theta}-D_{12} \frac{1}{a} \frac{\partial s}{\partial \theta}, i_{\phi}=\kappa E_{1 \phi}-D_{12} \frac{1}{a \sin \theta} \frac{\partial s}{\partial \phi}$,

where $\kappa$ is the surface conductivity, $D_{12}$ is the surface diffusion coefficient. By substituting Eqs. (9) and (10) into Eq. (8), we obtain

$\frac{\partial s}{\partial t}+\frac{s}{\tau_{\mathrm{s}}}-\frac{D_{12}}{a^{2}} \Delta_{\theta, \phi} s=$

$=\sigma_{1} \frac{\partial V_{1}}{\partial r}-\sigma_{\mathrm{b} 2} \frac{\partial V_{2}}{\partial r}+\frac{\kappa}{a^{2}} \Delta_{\theta, \phi} V_{1}, c c$,

where $\Delta_{\theta, \phi}$ denotes the spherical Laplace operator $\frac{1}{\sin \theta} \frac{\partial}{\partial \theta}\left(\sin \theta \frac{\partial}{\partial \theta}\right)+\frac{1}{\sin \theta^{2}} \frac{\partial^{2}}{\partial \phi^{2}}$.

For a sphere in a uniform electric field, Eq. (11) can be written in a simpler form:

$\frac{\partial s}{\partial t}+\frac{s}{\tau}=\sigma_{1} \frac{\partial V_{1}}{\partial r}-\sigma_{2} \frac{\partial V_{2}}{\partial r}$,

where $\tau_{\alpha}^{-1}=\tau_{\mathrm{s}}^{-1}+2 D_{12} / a^{2}$, and Eq. (2) holds. A contradiction arises here. The factor 2 staying in Eq. (2) indicates that the electric field is presumed to be uniform. This assumption was correct in O'Konski's study [40], but is unacceptable in the DEP research, when the electric field must be nonuniform $[7,8]$.

The above equation with $\partial s / \partial t=-i \omega s$ and $V_{1}, V_{2}$ defined by Eqs. (6) and (7) gives the following formula for the parameters of Eq. (3):

$s_{\mathrm{p}}=\varepsilon_{0}\left(\beta_{\mathrm{ld}}-\beta_{\mathrm{pd}}\right)\left(K_{2}+2 K_{1}\right) \Theta E$;

$E_{2}=\left[\frac{3 \sigma_{1}}{\sigma_{2}+2 \sigma_{1}} \Theta+\frac{3 K_{1}}{K_{2}+2 K_{1}}(1-\Theta)\right] E$,

where $\beta_{\text {ld }}=\left(\sigma_{2}-\sigma_{1}\right) /\left(\sigma_{2}+2 \sigma_{1}\right)$ is the ClausiusMossotti factor of a conductive sphere with $\sigma_{2}$ used instead of $\sigma_{\mathrm{b} 2}$, and $\beta_{\mathrm{pd}}=\left(K_{2}-K_{1}\right) /\left(K_{2}+2 K_{1}\right)$ is the Clausius-Mossotti factor of a perfect-dielectric sphere, $\Theta=\left[1+\tau_{\beta}\left(-i \omega+\tau_{\alpha}^{-1}\right)\right]^{-1}, \tau_{\beta}=\varepsilon_{0}\left(K_{2}+\right.$ $\left.+2 K_{1}\right) /\left(\sigma_{2}+2 \sigma_{1}\right)$.

Then cproposed general conductive-sphere model yields the following Clausius-Mossotti factor

$f_{\mathrm{CM}}=\Theta \beta_{\mathrm{ld}}+(1-\Theta) \beta_{\mathrm{pd}}$,

where $\beta_{\mathrm{ld}}=\left(\sigma_{2}-\sigma_{1}\right) /\left(\sigma_{2}+2 \sigma_{1}\right)$ is the ClausiusMossotti factor of a conductive sphere with $\sigma_{2}$ used

ISSN 2071-0194. Ukr. J. Phys. 2020. Vol. 65, No. 6 
instead of $\sigma_{\mathrm{b} 2}$, and $\beta_{\mathrm{pd}}=\left(K_{2}-K_{1}\right) /\left(K_{2}+2 K_{1}\right)$ is the Clausius-Mossotti factor of a perfect-dielectric sphere, $\Theta=\left[1+\tau_{\beta}\left(-i \omega+\tau_{\alpha}^{-1}\right)\right]^{-1}, \tau_{\beta}=\varepsilon_{0}\left(K_{2}+\right.$ $\left.+2 K_{1}\right) /\left(\sigma_{2}+2 \sigma_{1}\right)$. Equation (14) represents the electric field inside a weakly conductive dielectric sphere as a linear combination of the fields of the leaky dielectric and perfect-dielectric spheres. Similarly, Eq. (15) shows that the real part $\beta$ of the Clausius-Mossotti factor of a conductive sphere varies between two extreme values, $\beta_{\mathrm{ld}}$ and $\beta_{\mathrm{pd}}$. From Eqs. (13)-(15), we get the result of the TCM model [26] by setting $\tau_{\alpha}^{-1}=0$ and $\kappa=0$. If, in addition, we take $\omega=0$, the result of the original Taylor's study [23] is obtained.

\subsection{Polarizabilities of high orders}

To solve some problems, the potential of the external field source is assumed to have the following form $[55,56]$ :

$V_{0}=-\sum_{l, m} C_{l, m} r^{l} Y_{l}^{m}(\theta, \phi)$,

where $Y_{l}^{m}(\theta, \phi)$ is the spherical harmonic of degree $l$. Then the potential of the sphere at $r \geq a$ is

$V=\alpha_{l l, m} C_{l, m} r^{-l-1} Y_{l}^{m}(\theta, \phi)$,

where is the polarizability of order $l$. Using the proposed model, we get

$\alpha_{l}=\left[\Theta_{l} \beta_{l}^{(\mathrm{ld})}+\left(1-\Theta_{l}\right) \beta_{l}^{(\mathrm{pd})}\right] a^{2 l+1}$,

where

$\beta_{l}^{(\mathrm{ld})}=\frac{\sigma_{2 l}-\sigma_{1}}{\sigma_{2 l}+\sigma_{1}+\sigma_{1} / l}$,

$\beta_{l}^{(\mathrm{pd})}=\frac{K_{2}-K_{1}}{K_{2}+K s_{1}+K_{1} / l}$

$\Theta_{l}=\left[1+\tau_{\beta l}\left(-i \omega+\tau_{\alpha l}^{-1}\right)\right]^{-1}$,

$\tau_{\beta l}=\varepsilon_{0} \frac{K_{2}+K_{1}+K_{1} / l}{\sigma_{2 l}+\sigma_{1}+\sigma_{1} / l}$,

$\sigma_{2 l}=\sigma_{\mathrm{b} 2}+(l+1) \frac{\kappa}{a}$,

$\tau_{\alpha l}^{-1}=\tau_{\mathrm{s}}^{-1}+l(l+1) \frac{D_{12}}{a^{2}}$.

The term $l(l+1)$ comes from the spherical Laplace operator in the equation for the surface charge, Eq. (11). In our previous study of nonlocal effects in metals [57], the Laplace operator was present in a formula for the electric current. Similarly, Eqs. (19) show the dependences of the parameters $\sigma_{2 l}$ and $\tau_{\alpha l}$ on the field distribution that is taken into account with the parameter $l$. According to these equations, the higher the inhomogeneity of the electric field, the higher the significance of the surface processes. In particular, $\tau_{\beta l} / \tau_{\alpha l} \rightarrow \infty$ and, consequently, $\alpha_{l} \rightarrow \beta_{\mathrm{pd}} a^{2 l+1}$ at $l \rightarrow \infty$ or $a \rightarrow 0$. This property of $\alpha_{l}$ is likely to be important, when the expansions in spherical harmonics neglecting the surface charge transport converge slowly or diverge $[55,58,59]$.

\section{Extreme Values of the Clausius-Mossotti Factor}

The parameter $\tau_{\alpha}^{-1}$ introduced in Eq. (12) is the surface charge $\alpha$-relaxation rate defined as a sum of the surface-charge decay and diffusion rates, $\tau_{\mathrm{s}}^{-1}$ and $\tau_{\text {sd }}^{-1}=2 D_{12} / a^{2}$, respectively. The parameter $\tau_{\alpha}^{-1}$ has a significant influence on the form of the potential distribution. Below, we discuss some limiting cases focusing on transformations of the Clausius-Mossotti factor.

\subsection{Perfect-dielectric limit}

The absolute value of the factor $\Theta$ is small, $\Theta \simeq 0$, and Eq. (15) reduces to

$f_{\mathrm{CM}} \simeq \beta_{\mathrm{pd}}$

when

$\omega \tau_{\beta} \gg 1$ or $\tau_{\alpha} \ll \tau_{\beta}$.

If one of the above conditions is satisfied, we get the potential $V_{\text {pd }}$ defined in Table 1 . Hence, a sphere has properties of a perfect dielectric not only in a highfrequency ac field, but also at arbitrary $\omega$ including $\omega=0$, when the leakage of the surface charge is fast.

\subsection{Modified Taylor-Melcher leaky-dielectric limit}

The absolute value of the factor $1-\Theta$ is small, $1-\Theta \simeq$ $\simeq 0$, and Eq. (15) reduces to

$f_{\mathrm{CM}} \simeq \beta_{\mathrm{ld}}$

if

$\omega \tau_{\beta} \ll 1$ and $\tau_{\alpha} \gg \tau_{\beta}$. 


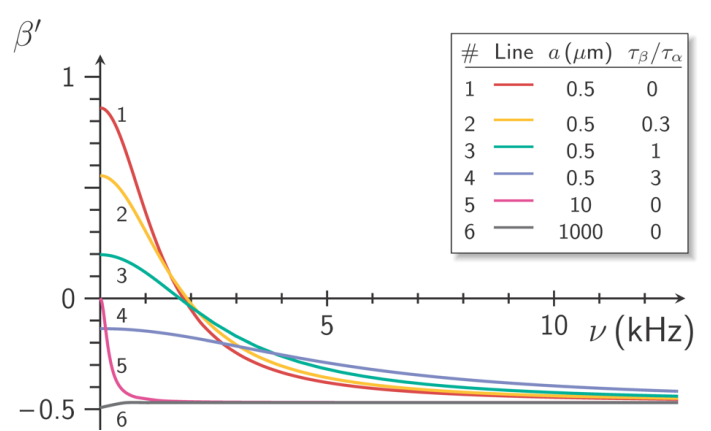

Fig. 1. Frequency dependence of the real part of the ClausiusMossotti factor calculated for polystyrene spheres in water

Hence, despite the common opinion $[2,7,27,28]$, the first condition (23) alone is not sufficient for the validity of the leaky-dielectric model. The potential $V_{\mathrm{ac}}$ of the ac leaky-dielectric model $[2,26]$ with $\sigma_{\mathrm{b} 2}$ replaced by $\sigma_{2}$ is obtained at $\omega \tau_{\alpha} \gg 1$.

\subsection{Surface conductor limit}

Let us assume that the following inequalities are satisfied: $\kappa / a \gg \sigma_{\mathrm{b} 2}$ and $\kappa / a \gg \sigma_{1}$. If so, both quantities $\sigma_{1}$ and $\sigma_{\mathrm{b} 2}$ can be omitted in Eqs. (13) and (15) which may be written as follows:

$s_{\mathrm{p}}=\frac{3 \varepsilon_{0} K_{1} E}{1+\vartheta}$

$f_{\mathrm{CM}}=\frac{1+\vartheta \beta_{\mathrm{pd}}}{1+\vartheta}$

$\vartheta=\left(-i \omega+\tau_{\alpha}^{-1}\right) \varepsilon_{0}\left(K_{2}+2 K_{1}\right) a / \kappa$. According to the above equation, the properties of a surface-conductor sphere can change in a broad range. Thus, at $\tau_{\alpha} \ll$ $\ll \varepsilon_{0}\left(K_{2}+2 K_{1}\right) a / \kappa$, we get

$s_{\mathrm{p}} \simeq 0$ and $f_{\mathrm{CM}} \simeq \beta_{\mathrm{pd}}$,

as for a perfect-insulator sphere. For $\omega=0$ and $\tau_{\alpha} \gg$ $\gg \varepsilon_{0}\left(K_{2}+2 K_{1}\right) a / \kappa$, we obtain the potential $V_{\mathrm{pc}}$ and other characteristics of a perfect-conductor sphere:

$s_{\mathrm{p}}=3 \varepsilon_{0} K_{1} E$

$f_{\mathrm{CM}}=\beta_{\mathrm{pc}}=1$.

Both perfect-dielectric and perfect-conductor models predict a diminution of the EHD flow, i.e. the tangential stress $S_{\theta}$ which is proportional to the product $s_{\mathrm{p}} E_{2}$ should be zero. According to the perfectdielectric model, $S_{\theta}=0$, because $s_{\mathrm{p}}=0$ at $E_{2} \neq 0$. On the contrary, the perfect-conductor model gives $s_{\mathrm{p}} \neq 0$; but $S_{\theta}=0$, since $E_{2}=0$.

\subsection{Frequency dependence of the Clausius-Mossotti factor}

To study how the polarization of a sphere depends on the parameter $\kappa_{12}$ and surface-charge $\alpha$-relaxation time, we consider Fig. 1. It presents the theoretical dependences of the factor $\beta$ on the frequency $\nu=\omega /(2 \pi)$ for polystyrene spheres immersed in water. The calculations were executed with $R=10 \mu \mathrm{m}$ and other parameters determined in experiments [29]. Figure 1 demonstrates a huge spread of the dc values of $\beta$, while, at high frequencies, all curves approach the perfect-dielectric limit. Comparing curves 1, 5 and 6 we see that properties of a small sphere with $2 a=1 \mu \mathrm{m}$ differ dramatically from those of large spheres. It is of interest that the micrometersized particle of a good insulator with $\sigma_{\mathrm{b} 1}=0$ can be considered at low frequencies as a good-conductor particle. Indeed, $\beta(\omega=0)=0.86$ of curve 1 is close to the ultimate value $\beta_{\mathrm{pc}}=1$ of the perfect conductor. The comparison of curves $1-4$ shows that values of $\beta_{\mathrm{dc}}$ decrease with increasing the ratio of the Maxwell-Wagner relaxation time $\tau_{\beta}$ to the surfacecharge $\alpha$-relaxation time $\tau_{\alpha}$. The cross-over frequency defined by the condition $\beta\left(\omega_{\mathrm{c}}\right)=0$ is a nonmonotonic function of $\tau_{\beta} / \tau_{\alpha}$.

From experiments, it is known that the DEP force can be changed by varying the frequency. If the factors $\beta_{\mathrm{pd}}$ and $\beta_{\mathrm{ld}}$ are of different signs, the cross-over frequency $[5-7,30,31,36,43,45]$ (or critical frequency [2]) can be introduced as the frequency dividing the regions of negative and positive dielectrophoreses at which the real part of the Clausius-Mossotti factor equals zero. From Eq. (15) and the definition of the parameter $\Theta$, we get

$\omega_{\mathrm{c}}=\tau_{\beta}^{-1}\left[\left(-\frac{\beta_{\mathrm{ld}}}{\beta_{\mathrm{pd}}}-\frac{\tau_{\beta}}{\tau}\right)\left(1+\frac{\tau_{\beta}}{\tau}\right)\right]^{1 / 2}$.

At $\tau_{\alpha}^{-1}=0$, the above equation gives the formula known in the literature $[4,6,36,43,45]$ :

$\omega_{\mathrm{c}}=\left[\frac{\sigma_{2}-\sigma_{1}}{\left(K_{1}-K_{2}\right) \varepsilon_{0}} \tau_{\beta}^{-1}\right]^{1 / 2}$.

According to Fig. 1 and Eq. (15), the factor $\beta=$ $=\Re f_{\mathrm{CM}}$ attains maximum at $\omega=0$, when the parameter $\Theta$ is equal to $\Theta_{0}=\left(1+\tau_{\beta} / \tau_{\alpha}\right)^{-1}$, and minimum at $\omega \rightarrow \infty$, when $\Theta=0$. The frequency

$\omega_{\mathrm{i}}=\tau_{\beta}^{-1}+\tau_{\alpha}^{-1}$, 
at which $\Re \Theta=\Theta_{0} / 2$, is called the inflection frequency [60].

\section{Experimental Evidences of Surface Conductivity}

\subsection{EHD fluid circulation}

A deformation and the fluid circulation in a dielectric drop embedded into an immiscible dielectric liquid in uniform ac and dc electric fields were theoretically and experimentally studied by Torza, Cox, and Mason [26]. They introduced a classification including three classes: class A at $q \leq Z$, class B at $0<q-Z<\delta Z(Z-1)^{2}$, and class $\mathrm{C}$ at $q-Z \geq$ $\geq \delta Z(Z-1)^{2}$, where $q=K_{2} / K_{1}, Z=\sigma_{2} / \sigma_{1}$, and $\delta=\left(5 \mu_{1}+5 \mu_{2} /\left(16 \mu_{1}+19 \mu_{2}\right) ; \mu_{1}\right.$ and $\mu_{2}$ are the viscosities of the ambient medium and a drop, respectively. The electrically induced flows on both sides of the interface are directed from the equator to the poles in systems of class $\mathrm{A}$ and from the poles to the equator in systems of classes $\mathrm{B}$ and $\mathrm{C}$. In systems of classes $\mathrm{A}$ and $\mathrm{B}$, the drop becomes a prolate spheroid. In systems of class $\mathrm{C}$, the drop is oblate at low frequencies and prolate at high frequencies.

According to Torza et al [26] and other researchers $[25,61]$, the types of deformation and flow directions changed to the opposite ones under the inversion. For example, pure silicone oil drops in castor oil were of class $\mathrm{C}$ and had an oblate deformation in a dc electric field. Castor-oil drops in silicone oil belonged to class A and were prolate. However, in one experiment, both a drop of polymethyl phenylsiloxane (PMPS) in spindle oil and a drops of spindle oil in PMPS were reported to stretch along the direction of the electric field [54] as for a system of class A.

Here, we propose an explanation for this result based on a proper account of the surface conductivity. If no other processes are taken into account, the extension of the TCM model reduces to a new definition of the parameter $Z$ :

$Z=\frac{R}{a}+\frac{\sigma_{\mathrm{b} 2}}{\sigma_{1}}$.

By increasing $\kappa$ or decreasing $a$, one can increase a value of $Z$. As a result, a system of class $\mathrm{C}$ can go to class B and then to class A.

The importance of the surface conductance was confirmed by experiments $[14,20]$ reporting the transition of a system of class $\mathrm{C}$ to class A. Thus, Mik- kelsen et al. [20] studied properties of separate millimeter-sized silicone oil drops suspended in castor oil in uniform dc or ac electric fields. Drops were covered by different spherical particles: silver coated hollow glass microspheres with average diameters of 15 and $55 \mu \mathrm{m}$, pure and two types of sulfonated polystyrene particles with a mean diameter of $40 \mu \mathrm{m}$. The electrical conductivities of the microbeads ranged from $10^{-11} \mathrm{~S} \mathrm{~m}^{-1}$ of polystyrene to $10^{7} \mathrm{~S} \mathrm{~m}^{-1}$ of silver. A pure silicone oil drop with $\varepsilon_{2}=2.8$ and $\sigma_{\mathrm{b} 2}=5^{-}$ $10 \mathrm{pS} \mathrm{m}^{-1}$ in castor oil with $\varepsilon_{1}=4.7$ and $\sigma_{1}=50$ $100 \mathrm{pS} \mathrm{m}^{-1}$ belongs to class $\mathrm{C}$ of the TCM classification. Indeed, the drop was oblate in the dc case and prolate in the ac case at a frequency $\nu=\omega /(2 \pi)$ of $200 \mathrm{~Hz}$, when $\tau_{\beta}$ was about $1 \mathrm{~s}$. Fluid flows were directed from the poles to the equator in agreement with the theory. The authors reported various processes including the drop deformation, fluid flows, migration of microparticles at the interface, and formation of surface structures. Drastic changes of drop's properties were observed in the dc field, when surface particles formed longitudinal chains spanning from pole to pole and thereby increasing $\kappa$. These were the oblate-to-prolate morphology transition and the reverse of the fluid flow, which confirms the transition of the system from TCM class $\mathrm{C}$ to class A. Other results of Ref. [20] are discussed below.

\subsection{DEP force}

The surface conductivity can change the sign of $\beta$ or $\beta^{\prime}$. In this case, the direction of the DEP force is changed, accordingly. Amah and co-authors [18] manipulated by microparticles absorbed at the surface of a millimeter-sized silicone-oil drop. They demonstrated the possibility to collect particles either on the poles or equator or to move the particles from the equator to the poles and vice versa. Experiments were performed with the use of silicone oil I from Dow Corning with $K_{2}=6.85$ and $\sigma_{\mathrm{b} 2}=56 \mathrm{pS} \mathrm{m}^{-1}$ and silicone oil II from United Chemical Technology with $K_{2}=2.75$ and $\sigma_{\mathrm{b} 2}=3.6 \mathrm{pS} \mathrm{m}^{-1}$. Castor oil with $K_{1}=4.7$ and $\sigma_{1}=32 \mathrm{pS} \mathrm{m}^{-1}$ was used as an ambient liquid. The particles trapped on drop's interface had approximately the spherical shape. Those made of soda lime glass with $K=6.9$ had diameters from 1 to $3 \mu \mathrm{m}$. The particles made of hollow glass with $K=1.2$ had diameters from 6 to $32 \mu \mathrm{m}$, and the particles of polystyrene with $K=2.0$ had a diameter of $4 \mu \mathrm{m}$. 


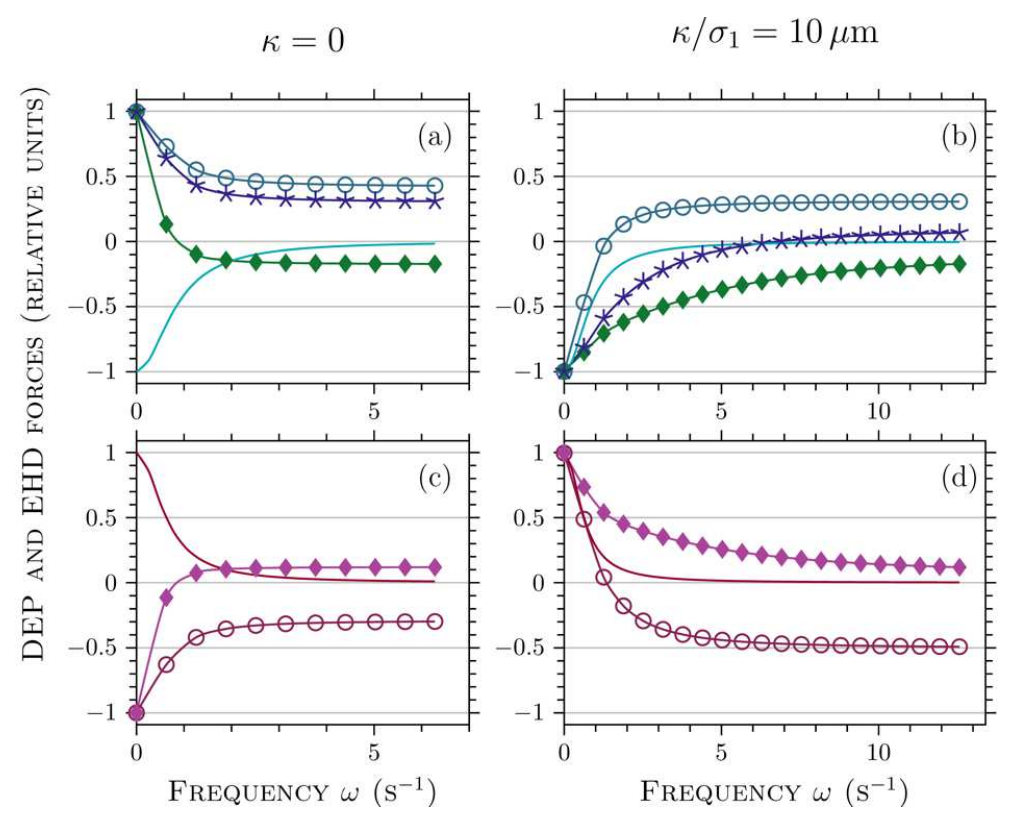

Fig. 2. Theoretical frequency dependences of the normalized EHD drag force (solid lines) and DEP force acting on microparticles of soda lime glass (lines with diamond marks), hollow glass (lines with circle marks), and polystyrene (lines with star marks) adsorbed on the surface of a silicone oil-I drop [ $(a)$ and $(b)]$ or silicone oil-II drop [ $(c)$ and $(d)]$ suspended in castor oil calculated neglecting $[(a)$ and $(c)]$ and allowing for $[(b)$ and $(d)]$ the surface conductivity of microparticles

It was of importance that Amah et al. [18] presumed that the DEP force was independent of the frequency and expressed through drop's $\beta_{\mathrm{pd}}$ and microparticle's $\beta_{\mathrm{pd}}^{\prime}$. The model of Torza, Cox and Mason was applied to determine the tangential stress that produces the fluid flow which drags the microparticles. The observed reversal of particles' velocities was explained by a competition between the EHD drag and DEP forces. Namely, these forces acting on the hollow glass and polystyrene microparticles at a dc electric field were assumed to be of the opposite directions with a stronger EHD drag. With increasing the field frequency, the tangential stress, the flow velocities, and EHD drag force decreased. Therefore, the DEP force should dominate at high-frequency ac fields. According to Amah et al., particles' velocities reversed at a critical frequency, when the EHD and DEP forces had equal amplitudes, but opposite directions.

In Fig. 2, the normalized EHD drag force $F_{\mathrm{EHD}}(\omega) /$ $/ F_{\mathrm{EHD}}(0)\left|=S_{\theta}(\omega) /\right| S_{\theta}(0) \mid$ is compared with the normalized DEP force [20]

$$
\frac{F_{\mathrm{DEP}}(\omega)}{\left|F_{\mathrm{DEP}}(0)\right|}=\frac{\beta(2+\beta) \beta^{\prime}}{\left|\beta_{\mathrm{ld}}\left(2+\beta_{\mathrm{ld}}\right) \beta_{\mathrm{ld}}^{\prime}\right|} .
$$

Left-column figures 2, $a$ and 2, $c$ present the forces calculated with the original Taylor-Melcher leakydielectric model, and right-column figures $2, b$ and $2, d$ show the results of the model allowing for the surface conductivity. This figure reveals a mistake in the previous theoretical model. Contrary to Ref. [18], the frequency dependence of the DEP force can be more sharp than that of the EHD drag force in the region of $0<\omega<10 \mathrm{~s}^{-1}$, and $F_{\mathrm{DEP}}(\omega)$ can even change its sign. If this mistake is corrected, and the original leaky-dielectric model is used, the theory will disagree with experiment. Namely, Amah and co-authors [18] noted that the DEP force and EHD force acting on glass particles in drops of both silicone oil I and silicone oil II were always of the same direction. These experimental findings are in the qualitative disagreement with the results of calculations of $F_{\mathrm{DEP}}(\omega)$ presented in Figs. 2, $a$ and 2, $c$. This contradiction can be resolved by accounting for the surface conductivity of particles $\kappa$ estimated by us as $\kappa=\sigma_{1} \times 10 \mu \mathrm{m}$. However, it is the correction that leads to a dramatic change in the interpretation of the experimental results. Indeed, according to Figs. $2, b$ and $2, d$, the EHD and DEP forces are always of the same direction. So, there is no competition between the forces. 
According to our calculations presented in Fig. 2, $b$, the DEP force acting on a polystyrene sphere with a diameter of $4 \mu \mathrm{m}$ changes the sign at an angular frequency of $\omega_{0}=6.8 \mathrm{~s}^{-1}$ determined by formula (30). This value should be smaller than the critical angular frequency $\omega_{\mathrm{c}}$ at which the microparticles change the direction of motion because of the presence of the opposite EHD drag. In the experiment [18], the value of $\omega_{\mathrm{c}}$ was determined to be of the order of $10 \mathrm{~s}^{-1}$, i.e. the theoretical $\omega_{0}$ is about the experimental $\omega_{\mathrm{c}}$. In this case, the EHD drag could be neglected. In the experiment with hollow glass particles of different sizes from 6 to $32 \mu$ the change in the direction of motion was observed at frequencies decreasing with the size [18]. Namely, $\omega_{\mathrm{c}}$ was about $5 \mathrm{~s}^{-1}$ for the smallest particles, while $\omega_{\mathrm{c}}$ was theoretically estimated to be $2.1 \mathrm{~s}^{-1}$ for the particles of the average size equal to $18 \mu \mathrm{m}$. We find $\omega_{0}=4.2 \mathrm{~s}^{-1}$ very close to the experimental $\omega_{\mathrm{c}}$ for the smallest hollow glass particles and $\omega_{0}=1.3 \mathrm{~s}^{-1}$ for the average-size ones. Additional arguments in favor of the proposed model can be found in Ref. 62 .

\section{Experimental Evidences of $\alpha$-Relaxation}

\subsection{EHD fluid circulation}

In the research by Dommersnes et al. [14], the clay clusters were dispersed throughout a millimeter-sized silicone oil drop at concentrations of about $1 \mathrm{wt} \%$. In $\mathrm{dc}$ and low-frequency ac electric fields, the chains of highly conducting clusters were formed along the field direction at the surface of a silicone oil drop immersed in castor oil. At sufficiently high fields and clay concentrations, the drop deformation was observed to change from oblate to prolate. Simultaneously, the EHD flow was suppressed. According to the conclusion of the previous subsection, there are two ways to explain this result. The first way is to use the perfectdielectric model, as was, in fact, done by Dommersnes and co-authors [14] who concluded that the strongly enhanced surface conductivity should diminish the charge buildup on the drop and thereby suppress the EHD flow. However, this model does not explain the stretching and breakdown of a silicone-oil drop covered by highly conductive microparticles, which was observed in Ref. [20].

According to Mikkelsen et al. [20], at a sufficiently strong dc field of $200 \mathrm{~V} \mathrm{~mm}^{-1}$ and a high particle coverage of $0.6-0.8$, chains of highly conducting mi-

ISSN 2071-0194. Ukr. J. Phys. 2020. Vol. 65, No. 6 croparticles connected drop's poles. In this extreme case, the drop is elongated and broken apart, whereas the EHD fluid flows are suppressed. The authors of the research inferred that, in a dc field, "the particle chains redistribute the free charges at the drop interface until the electric field component, which is parallel to the drop surface, becomes zero". This statement is in complete agreement with our engagement of the perfect-conductor model.

\subsection{DEP force}

There were a few experiments which reported on an increase of the cross-over frequency with decreasing the particle radius. For instance, Ermolina and Morgan [43] reported on the cross-over frequencies $\nu_{\mathrm{c}}=\omega_{\mathrm{c}} /(2 \pi)$ for polystyrene latex beads suspended in aqueous $\mathrm{KCl}$ solutions with conductivities ranging from $10^{-4}$ to $10^{-1} \mathrm{~S} / \mathrm{m}$. By using Eq. (30), they determined the surface conductance $\kappa$ falling in the range 0.9 to $1.2 \mathrm{nS}$ for particles with diameters from 110 to $1900 \mathrm{~nm}$ and $\kappa=0.75 \mathrm{nS}$ for particles with a diameter of $44 \mathrm{~nm}$.

Let us consider the formula of the cross-over Eq. (29), by assuming that the particles are extremely small, so that the surface conductance dominates on the right-hand side of Eq. (2): $\beta_{\text {ld }}$ approaches unity, and the term $2 D_{12} / a^{2}$ dominates in the definition of $\tau_{\alpha}^{-1}$. Under these conditions, Eq. (29) takes the form

$\omega_{\mathrm{c}}=\frac{2 \kappa}{\varepsilon_{0}\left(K_{2}+2 K_{1}\right)} \frac{1}{a}\left[\left(\frac{1}{\beta_{\mathrm{pd}}}-\frac{L}{a}\right)\left(1+\frac{L}{a}\right)\right]^{1 / 2}$,

where $L=D_{12}\left(K_{2}+2 K_{1}\right) \varepsilon_{0} / \kappa$. According to Fig. 3, the above equation is in good agreement with the experimental data taken from Fig. 1 of Ref. [43] at $\sigma_{1}=0.7 \times 10^{-4} \mathrm{~S} / \mathrm{m}$. Here, the quantities $\kappa$ and $D_{12}$ are considered as adjustable parameters. A fit gave $\kappa=0.91 \mathrm{nS}$ and $D_{12}=1.8 \times 10^{-8} \mathrm{~m}^{2} / \mathrm{s}$. The straight dotted line drawn, by neglecting the surface diffusion, is presented for comparison. As seen from Fig. 3, a better fit is obtained, when the surface diffusion is taken into account. At that, the solid line crosses the experimental marks when $1 / a=9.3$ and $18.2 \mu \mathrm{m}^{-1}$ above the straight line and falls below this line at $1 / a \geq 45 \mu \mathrm{m}^{-1}$. Despite the good agreement between the experiment and the theory, Fig. 3 illustrates the dependence of the cross-over frequency on 


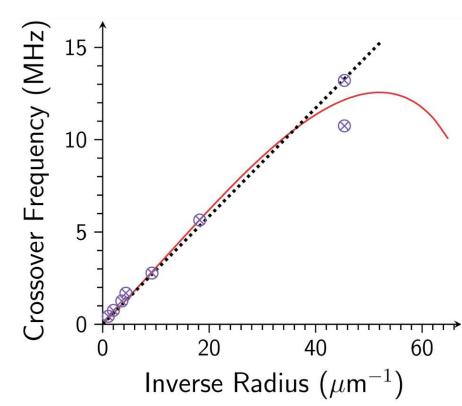

Fig. 3. Cross-over frequencies $\nu_{\mathrm{c}}=\omega_{\mathrm{c}} /(2 \pi)$ measured for ultra-tin polystyrene microparticles of various sizes in an aqueous $\mathrm{KCl}$ solution (marks) and their theoretical fits by Eq. (34) with $\kappa=0.91 \mathrm{nS}, D_{12}=1.8 \times 10^{-8} \mathrm{~m}^{2} / \mathrm{s}$ (solid line) and $D_{12}=0$ (dotted line)

the parameter $1 / R$, but the estimated value of $D_{12}$ may be incorrect. This value of $D_{12}$ is much higher than diffusivities of both cations and anions in bulk electrolytes [32, 34, 45, 63, 64], $1.96 \times 10^{-9} \mathrm{~m}^{2} \mathrm{~s}^{-1}$ according to Ref. [64] or $5 \times 10^{-9} \mathrm{~m}^{2} \mathrm{~s}^{-1}$ according to Refs. $[34,45]$ for $\mathrm{K}^{+}$and $\mathrm{Cl}^{-}$. A lower value of $D_{12}$ will be obtained, if one considers additional mechanisms of surface-charge dissipation, for example, the charge diffusion normal to the interface.

\section{Conclusion}

We have derived a general formula for the potential distribution inside and outside a conductive sphere in a uniform electric field allowing for the interfacial conductivity and the $\alpha$-relaxation of the surface charge due to the interfacial diffusion and the charge emission from the interface. This formula yields the known potentials of the leaky-dielectric, perfect-dielectric, and perfect-conductor models in limiting cases. In addition, we have defined a new surface-conductor limit that should be of particular importance in microfluidics. The theoretical description of all the states of a polarized sphere beyond the leaky-dielectric model is in accord with recent experiments.

We emphasize that, in the dc case, the leakydielectric model is valid, only if the surface relaxation time $\tau_{\alpha}$ is much larger than the Maxwell-Wagner relaxation time $\tau_{\beta}, \tau_{\alpha} \gg \tau_{\beta}$, while, at $\tau_{\alpha} \ll \tau_{\beta}$ and $\omega \simeq 0$, the perfect dielectric model is applicable contrary to the common concept. The last model can also be used at high frequencies, when $\omega \tau_{\beta} \gg 1$, and arbitrary $\tau_{\alpha}$.

Experimental evidences demonstrating the importance of the surface conductivity and the surface- charge $\alpha$-relaxation of a drop and embedded microparticles in the DEP and EHD processes have been presented. The tangential Maxwell stress at the surface of a weakly conducting sphere, or EHD drag force, has been shown to vanish at high and low values of the $\alpha$-relaxation rate introduced in this paper. It is worth noting that both these cases were observed in a silicone oil drop suspended in castor oil, when the surface was covered by highly conducting microparticles [14, 20]. Lamellar clay microparticles with sharp edges transformed the conductive drop into a perfect dielectric [14]. While, under similar conditions, silver-coated hollow glass microspheres and sulfonated polystyrene microbeads enabled the perfect-conductor limit to be achieved [20].

The current understanding of the experiment on a manipulation by microparticles attached to the interface of a silicone-oil drop with varying the frequency of the electric field [18] has been shown to be misleading. Due to the surface conductivity of the microparticles, at zero or low frequencies, the electrohydrodynamic (EHD) drag and dielectrophoretic (DEP) forces are in the same direction, but not opposite, as stated previously. The motion of microparticles turns back, because of the reversal of the DEP force with varying the field frequency. The surface conductivity of a drop can also explain experiments, in which the prolate deformation persists after liquids of the drop and the ambient medium are interchanged [54]. The model allowing for the diffusion of a surface charge has been found to be in good agreement with the experimental dependence of the cross-over frequency on the size of ultra-tin microparticles [43].

We have shown that the surface-charge transport processes depend on the angular distribution of an electric field. Namely, it has been established a quadratic dependence of the surface-charge diffusion rate and a linear dependence of the total conductivity of a sphere on the degree of the spherical harmonic of the external electric field. This effect can be important in systems of closely spaced particles and in the DEP process.

This work was supported by the Ministry of Education and Science of Ukraine, project 19BF051-01.

1. N.G. Green, H. Morgan. Separation of submicrometre particles using a combination of dielectrophoretic and electrohydrodynamic forces. J. Phys. D 31, L25 (1998).

ISSN 2071-0194. Ukr. J. Phys. 2020. Vol. 65, No. 6 
2. T.B. Jones. Electromechanics of Particles (Cambridge Univ. Press, 1995) [ISBN: 9780521019101].

3. A.V. Delgado. Interfacial Electrokinetics and Electrophoresis (Dekker, 2001) [ISBN: 0-8247-0603-X].

4. M.P. Hughes. Nanoelectromechanics in Engineering and Biology (CRC Press, 2003) [ISBN: 0-8493-1183-7].

5. H.-C. Chang, L. Yeo. Electrokinetically driven Microfluidics and Nanofluidics (Cambridge Univ. Press, 2010) [ISBN: 9780521860253].

6. A. Ramos. Electrokinetics and Electrohydrodynamics in Microsystems (Springer, 2011) [ISBN: 978-3-7091- 0899-4].

7. B. Cetin, D. Li. Dielectrophoresis in microfluidics technology. Electrophoresis 32, 2410 (2011).

8. T.Z. Jubery, S.K. Srivastava, P. Dutta. Dielectrophoretic separation of bioparticles in microdevices: A review. Electrophoresis 35, 691 (2014).

9. R.R. Pethig. Dielectrophoresis: Theory, Methodology and Biological Applications (Wiley, 2017) [ISBN: 9781118671450].

10. Q. Chen, Y.J. Yuan. A review of polystyrene bead manipulation by dielectrophoresis. RSC Adv. 9, 4963 (2019).

11. S. Nudurupati, M. Janjua, N. Aubry, P. Singh. Concentrating particles on drop surfaces using external electric fields. Electrophoresis 29, 1164 (2008),

12. S. Nudurupati, M. Janjua, P. Singh, N. Aubry. Effect of parameters on redistribution and removal of particles from drop surfaces. Soft Mat. 6, 1157 (2010).

13. P.F. Salipante, P.M. Vlahovska. Electrohydrodynamics of drops in strong uniform dc electric fields. Phys. Fluids 22, 112110 (2010)

14. P. Dommersnes, Z. Rozynek, A. Mikkelsen, R. Castberg, K. Kjerstad, K. Hersvik, J.O. Fossum. Active structuring of colloidal armour on liquid drops. Nat. Comm. 4, 2066 (2013).

15. Z. Rozynek, P. Dommersnes, A. Mikkelsen, L. Michels, J. Fossum. Electrohydrodynamic controlled assembly and fracturing of thin colloidal particle films confined at drop interfaces, Eur. Phys. J. Spec. Top. 223, 1859 (2014).

16. H. Yan, L. He, X. Luo, J. Wang, X. Huang, Y. Lü, D. Yang. Investigation on transient oscillation of droplet deformation before conical breakup under alternating current electric field. Langmuir 31, 8275 (2015).

17. R. Vaidyanathan, S. Dey, L.G. Carrascosa, M.J.A. Shiddiky, M. Trau. Alternating current electrohydrodynamics in microsystems: Pushing biomolecules and cells around on surfaces. Biomicrofluidics 9, 061501 (2015).

18. E. Amah, K. Shah, I. Fischer, P. Singh. Electro- hydrodynamic manipulation of particles adsorbed on the surface of a drop. Soft Mat. 12, 1663 (2016).

19. Q. Brosseau, P. M. Vlahovska. Streaming from the equator of a drop in an external electric field. Phys. Rev. Lett. 119 , 034501 (2017).

20. A. Mikkelsen, K. Khobaib, F.K. Eriksen, K.J. Maloy, Z. Rozynek. Particle-covered drops in electric fields: drop deformation and surface particle organization. Soft Mat. 14, 5442 (2018).
21. P.M. Vlahovska. Electrohydrodynamics of drops and vesicles. Ann. Rev. Fluid Mech. 51, 305 (2019).

22. L. Novotny, B. Hecht. Principles of nano-optics (Cambridge Univ. Press, 2006) [ISBN: 978-0-511-16811-6].

23. G. Taylor. Studies in electrohydrodynamics. I. The circulation produced in a drop by electrical field. Proc. Roy. Soc. Lond. A 291, 159 (1966).

24. J.R. Melcher, G.I. Taylor. Electrohydrodynamics: A review of the role of interfacial shear stresses. Ann. Rev. Fluid Mech. 1, 111 (1969).

25. D.A. Saville. Electrohydrodynamics: The Taylor- Melcher leaky dielectric model. Ann. Rev. Fluid Mech. 29, 27 (1997).

26. S. Torza, R.G. Cox, S.G. Mason. Electrohydrodynamic deformation and burst of liquid drops. Phil. Trans. R. Soc. Lond. A 269, 295 (1971).

27. R. Pethig. Dielectrophoresis: Status of the theory, technology, and applications. Biomicrofluidics 4, 022811 (2010).

28. B.A. Kemp, C.J. Sheppard. Field and material stresses predict observable surface forces in optical and electrostatic manipulation. Proc. SPIE 9922, 9922 (2016).

29. W. Arnold, H. Schwan, U. Zimmermann. Surface conductance and other properties of latex particles measured by electrorotation J. Phys. Chem. 91, 5093 (1987).

30. L. Gorre-Talini, S. Jeanjean, P. Silberzan. Sorting of brownian particles by the pulsed application of an asymmetric potential. Phys. Rev. E 56, 2025 (1997).

31. M.P. Hughes, H. Morgan, M.F. Flynn. The dielectrophoretic behavior of submicron latex spheres: Influence of surface conductance. J. Coll. Int. Sci. 220, 454 (1999).

32. M. Jiménez, F. Arroyo, F. Carrique, U. Kaatze, A. Delgado. Determination of stagnant layer conductivity in polystyrene suspensions: temperature effects. J. Coll. Int. Sci. 281, 503 (2005).

33. A. Delgado, F. González-Caballero, R. Hunter, L. Koopal, J. Lyklema. Measurement and interpretation of electrokinetic phenomena. J. Coll. Int. Sci. 309, 194 (2007).

34. S. Basuray, H.-C. Chang. Induced dipoles and dielectrophoresis of nanocolloids in electrolytes. Phys. Rev. E 75, 060501 (2007).

35. M.D. Vahey, J. Voldman. High-throughput cell and particle characterization using isodielectric separation. Anal. Chem. 81, 2446 (2009).

36. S. Basuray, H.-C. Chang. Designing a sensitive and quantifiable nanocolloid assay with dielectrophoretic crossover frequencies. Biomicrofluidics 4, 013205 (2010).

37. T. Honegger, K. Berton, E. Picard, D. Peyrade. Determination of Clausius-Mossotti factors and surface capacitances for colloidal particles. Appl. Phys. Lett. 98, 181906 (2011).

38. P.-Y. Weng, I.-A. Chen, C.-K. Yeh, P.-Y. Chen, J.-Y. Juang. Size-dependent dielectrophoretic crossover frequency of spherical particles. Biomicrofluidics 10, 011909 (2016).

39. H.P. Schwan. Electrical properties of tissue and cell suspensions. Adv. Biol. Med. Phys. 5, 147 (1957).

40. C.T. O'Konski. Electric properties of macromolecules. V. Theory of ionic polarization in polyelectrolytes. J. Phys. Chem. 64, 605 (1960). 
41. G. Schwarz. A theory of the low-frequency dielectric dispersion of colloidal particles in electrolyte solution. J. Phys. Chem. 66, 2636 (1962).

42. S. Tsukahara, T. Sakamoto, H. Watarai. Positive dielectrophoretic mobilities of single microparticles enhanced by the dynamic diffusion cloud of ions. Langmuir 16, 3866 (2000).

43. I. Ermolina, H. Morgan. The electrokinetic properties of latex particles: Comparison of electrophoresis and dielectrophoresis. J. Coll. Int. Sci. 285, 419 (2005).

44. M.-T. Wei, J. Junio, aH. D. Ou-Yang. Direct measurements of the frequency-dependent dielectrophoresis force. Biomicrofluidics 3, 012003 (2009).

45. S. Basuray, H.-H. Wei, H.-C. Chang. Dynamic double layer effects on ac-induced dipoles of dielectric nanocolloids. Biomicrofluidics 4, 022801 (2010).

46. C.-K. Yeh, J.-Y. Juang,.Dimensional analysis and prediction of dielectrophoretic crossover frequency of spherical particles. AIP Adv. 7, 065304 (2017).

47. K.K. Rangharajan, S. Prakash. Surface-modified microfluidics and nanofluidics. In: Encyclopedia of Nanotechnology (Springer Netherlands, 2014).

48. N.G. Green, H. Morgan. Dielectrophoresis of submicrometer latex spheres. 1. Experimental results. J. Phys. Chem. $B$ 103, 41 (1999).

49. L.A. Rosen, D.A. Saville. Dielectric spectroscopy of colloidal dispersions: Comparisons between experiment and theory. Langmuir 7, 36 (1991).

50. S.S. Dukhin, V.N. Shilov. Dielectric Phenomena and Double Layer in Disperse Systems and Polyelectrolytes (Naukova Dumka, 1972) (in Russian).

51. A. Korzhenko, M. Tabellout, J. Emery. Dielectric relaxation properties of the polymer coating during its exposition to water. Mat. Chem. Phys. 65, 253 (2000).

52. J. Lyklema, A. de Keizer, B.H. Bijsterbosch, G.J. Fleer, M.A. Cohen Stuart. Fundamentals of Interface and Colloid Science. Volume 2: Solid-Liquid Interfaces (Academic Press, 1995) [ISBN: 0-12-460521-9].

53. J. Lyklema, M. Minor. On surface conduction and its role in electrokinetics. Coll. Surf. A 140, 33 (1998).

54. A.I. Zhakin. Electrohydrodynamics of charged surfaces. Phys.-Uspekhi 56, 141 (2013).

55. R. Fuchs, F. Claro. Multipolar response of small metallic spheres: Nonlocal theory. Phys. Rev. B 35, 3722 (1987).

56. E. Bichoutskaia, A.L. Boatwright, A. Khachatourian, A.J. Stace. Electrostatic analysis of the interactions between charged particles of dielectric materials. J. Chem. Phys. 133, 024105 (2010).

57. V.V. Datsyuk, O.R. Pavlyniuk. Properties of longitudinal electromagnetic oscillations in metals and their excitation at planar and spherical surfaces. Nanoscale Res. Lett. 12, 473 (2017).

58. V.V. Datsyuk, O.M. Tovkach. Optical properties of a metal nanosphere with spatially dispersive permittivity. J. Opt. Soc. Am. B 28, 1224 (2011).

59. E.B. Lindgren, H.-K. Chan, A. J. Stace, E. Besley. Progress in the theory of electrostatic interactions between charged particles. Phys. Chem. Chem. Phys. 18, 5883 (2016).
60. H. Watarai, T. Sakamoto, S. Tsukahara. In Situ measurement of dielectrophoretic mobility of single polystyrene microparticles. Langmuir 13, 2417 (1997).

61. T. Tsukada, T. Katayama, Y. Ito, M. Hozawa. Theoretical and experimental studies of circulations inside and outside a deformed drop under a uniform electric field. J. Chem. Eng. Japan 26, 698 (1993).

62. V.V. Datsyuk, O.R. Pavlyniuk. The role of surface conductivity in electro-mechanics of microparticles in a meaklyconducting dielectric drop, in 2019 IEEE 39th International Conference on Electronics and Nanotechnology (ELNANO, 2019).

63. G. Supeene, C.R. Koch, S. Bhattacharjee. Deformation of a droplet in an electric field: Nonlinear transient response in perfect and leaky dielectric media. J. Coll. Int. Sci. 318, 463 (2008).

64. Y.-W. Liu, S. Pennathur, C.D. Meinhart. Electrophoretic mobility of a spherical nanoparticle in a nanochannel. Phys. Fluids 26, 112002 (2014).

Received 06.02.20

\section{В.В. Дацюк, О.Р. Павлинюк}

\section{РОЛЬ ТРАНСПОРТУ ПОВЕРХНЕВОГО} ЗАРЯДУ В ЕЛЕКТРОГІДРОДИНАМІЦІ ТА ЕЛЕКТРОМЕХАНІЦ ДІЕЛЕКТРИЧНОЇ КУЛІ

$\mathrm{P}$ е $з$ ю м е

Для моделювання електрокінетичних процесів у слабопровідних діелектричних середовищах широко використовують модель неідеального діелектрика Тейлора-Мелчера, хоча умови $\dddot{11}$ застосування невідомі. Для визначення цих умов знайдено розподіл електричного потенціалу всередині і зовні діелектричної кулі в електричному полі у припущенні, що куля і зовнішнє середовище є поганими провідниками струму, а також із врахуванням електричних i дифузійних поверхневих струмів та розпаду поверхневого заряду. Раніше будь-яка характеристика електричного поля діелектричної кулі, зокрема, реальна частина фактора Клаузіуса-Моссотті, знайдена для поля сталого струму, як правило, вважалася однозначною функцією двох параметрів, а саме, провідностей кулі та зовнішнього середовища. У нас ця величина залежить від більшої кількості параметрів i, у випадку сталого струму, може змінюватися від значення, визначеного для ідеального-діелектрика, до значення ідеально провідника, навіть для частинки гарного ізолятора. Використовуючи пропоновану теорію, різноманітні експериментальні результати щодо електрогідродинамічної (ЕГД) циркуляції рідини та діелектрофорезу (ДЕФ) мікрочастинок у діелектричних краплях ми пояснюємо вперше або по-новому. Частоти інверсії та перегину діелектрофорезу визначено з урахуванням затухання поверхневого заряду. Вперше передбачено залежність ефективної провідності кулі від кутового розподілу поля.

ISSN 2071-0194. Ukr. J. Phys. 2020. Vol. 65, No. 6 